measures. We aim to summarize an elaborated list of gut microbiome diversity measures.

Methods Systematic search was conducted in three databases: PubMed, Embase, and Cochrane Central Register of Clinical Trials for randomized controlled trials, quasi-experimental and observational studies for the relationship between gut microbiota and metabolic diseases published in 2019 with the English language.

Results The measurement methods of alpha diversity and beta diversity were explored. Of 5929 potential studies, 47 were included in the systematic review (14632 patients). Of 13 alpha diversity measures, the Shannon index was the most commonly used in 37 studies (78.7\%), followed by Chao1 index (19 studies), Operational Taxonomic Unit (OTU) richness (15 studies), Simpson index (13 studies), and Abundance-based Coverage Estimators (ACE) index (10 studies). Of 2 beta diversity measures, the UniFrac was the most commonly used in 24 studies (unweighted 17 studies and weighted 16 studies), followed by Bray-Curtis dissimilarity (16 studies).

Conclusions Various measurements of gut microbiome diversity have been used in the literature. All measurements have unique characteristics, advantages, and disadvantages which lead to different usage frequencies. The measures were chosen considering cost, simplicity, and types of research.

\section{IDDF2021-ABS-0106 SIGNIFICANCE OF THE EXPRESSION OF IRON DEATH-RELATED GENE HSBP1 IN ESOPHAGEAL SQUAMOUS CELL CARCINOMA}

Mingxin Zhang ${ }^{*}$, Ning Lu, Qian Li, Manli Cui. The First Affiliated Hospital of Xi'an Medical University, China

\subsection{6/gutjnl-2021-IDDF.38}

Background To analyze the prognosis and expression of the ferroptosis-related gene HSBP1 in esophageal squamous cell carcinoma and its possible regulatory mechanisms.
Methods Screen ferroptosis-related genes from previously published literature and GSEA (Gene Set Enrichment Analysis) website. We used R 4.0.3 software to perform differential analysis on the expression and prognosis of ferroptosis-related genes, and to evaluate the immune score.

Results A total of 30 ferroptosis-related genes were differentially expressed in esophageal squamous cell carcinoma (IDDF2021-ABS-0106 Figure 1, IDDF2021-ABS-0106 Figure 2). Prognostic analysis showed that only HSBP1 was associated with the prognosis of esophageal squamous cell carcinoma, and its high expression indicated a poor prognosis (IDDF2021-ABS-0106 Figure 3). In addition, univariate and multivariate COX regression analysis further proved that high expression of HSBP1 is an independent risk factor affecting prognosis (IDDF2021-ABS-0106 Table 1). GSEA enrichment

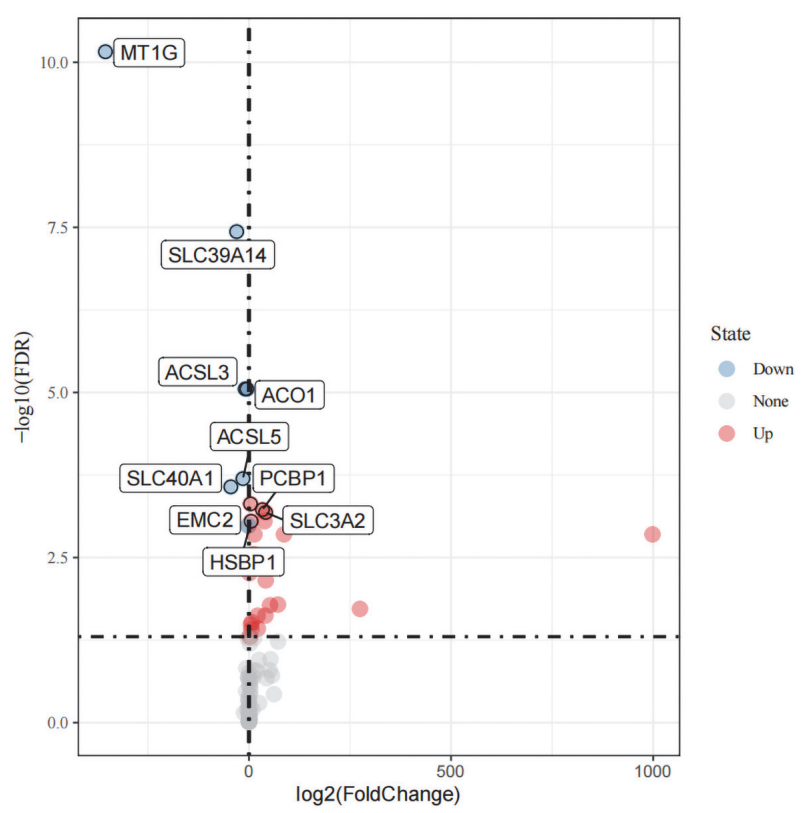

Abstract IDDF2021-ABS-0106 Figure 2

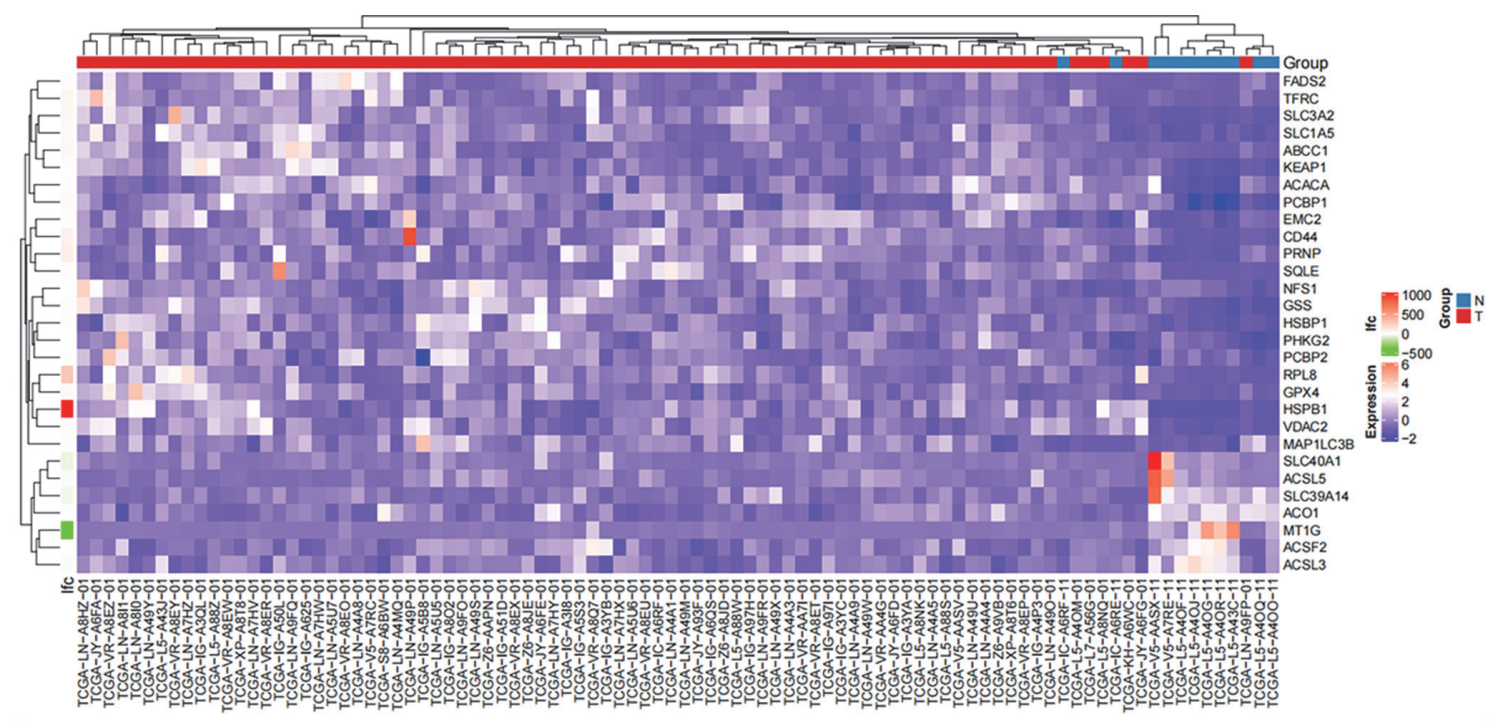




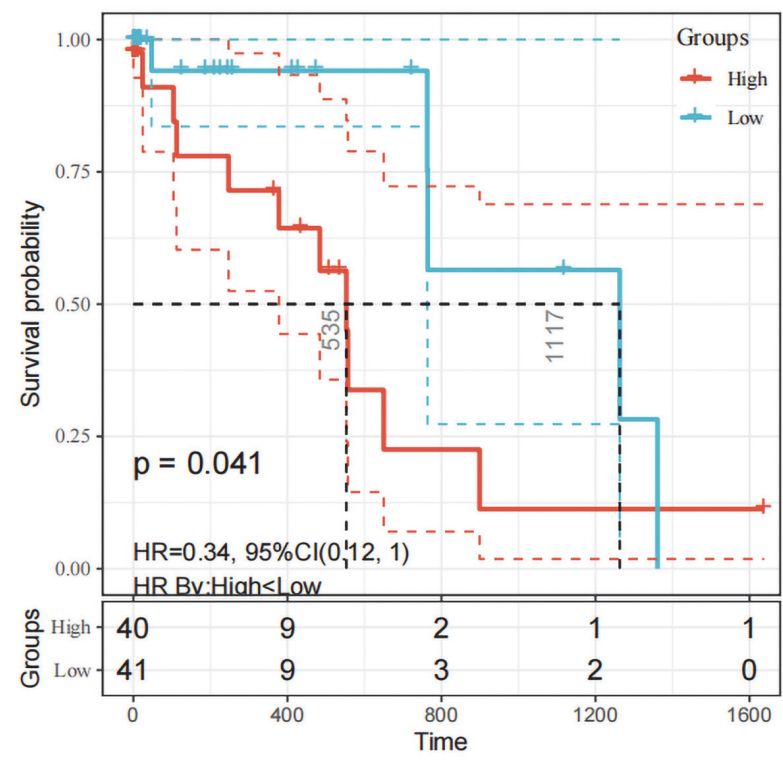

Abstract IDDF2021-ABS-0106 Figure 3
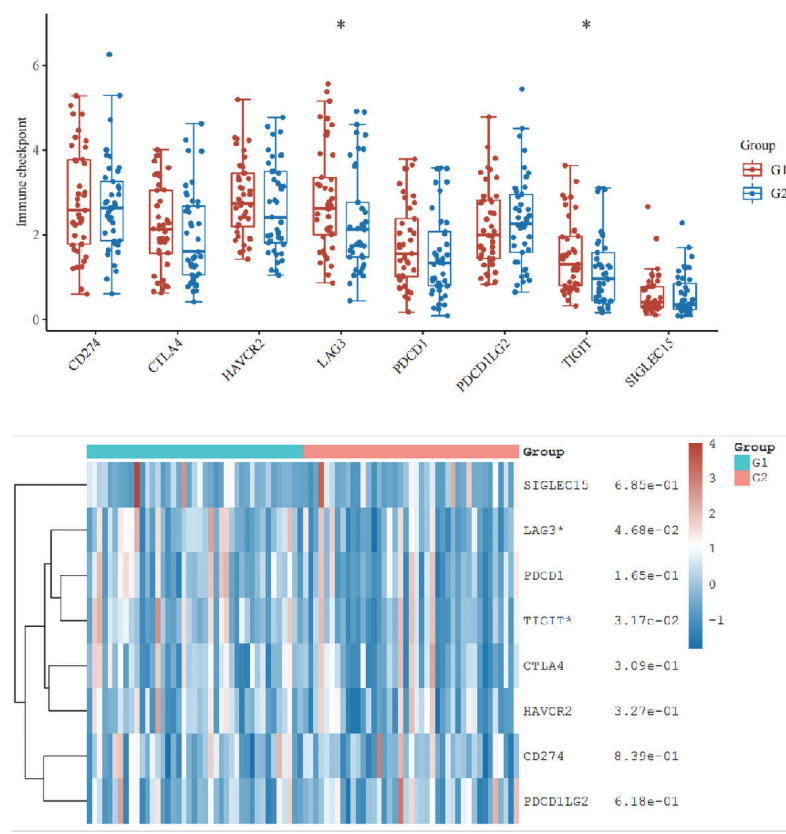

Abstract IDDF2021-ABS-0106 Figure 5
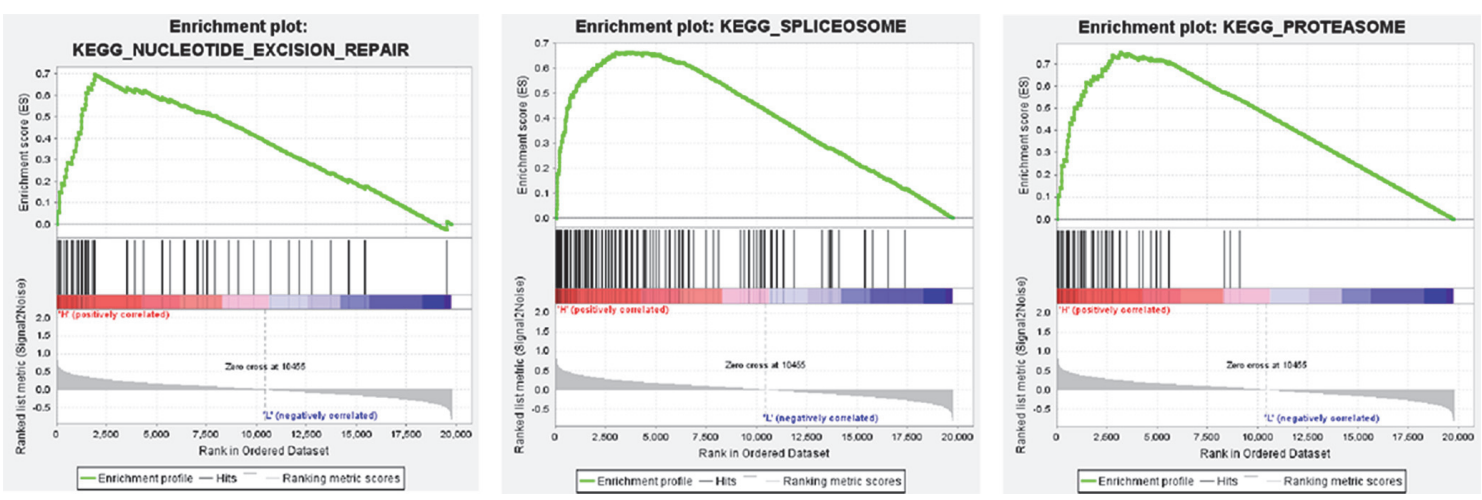

Abstract IDDF2021-ABS-0106 Table 1 COX regression analysis of univariate and multivariate factors affecting the prognosis of esophageal squamous cell carcinoma

\begin{tabular}{|c|c|c|c|c|c|c|}
\hline & \multicolumn{3}{|c|}{ Univariate analysis } & \multicolumn{3}{|c|}{ Multivariate analysis } \\
\hline & HR $(95 \% \mathrm{Cl})$ & $x^{2}$ & $P$ & HR $(95 \% \mathrm{Cl})$ & $x^{2}$ & $P$ \\
\hline HSBP1 & & 4.191 & 0.041 & & 6.23 & 0.01 \\
\hline High expression & Ref & & & Ref & & \\
\hline Low expression & $0.34(0.12-1.00)$ & & & $0.56(0.35-0.88)$ & & \\
\hline Gender & & 4.869 & 0.027 & & 6.24 & 0.01 \\
\hline Male & Ref & & & Ref & & \\
\hline Female & $0.10(0.01-0.78)$ & & & $\begin{array}{l}3.79 \\
(1.33-10.77)\end{array}$ & & \\
\hline Age. years & & 1.620 & 0.203 & & & \\
\hline$\leq 60$ & Ref & & & & & \\
\hline$>60$ & $1.76(0.74-4.20)$ & & & & & \\
\hline Race & & 4.346 & 0.114 & & & \\
\hline Asian & Ref & & & & & \\
\hline Whites & $1.26(0.49-3.26)$ & & & & & \\
\hline Black and others & $\begin{array}{l}3.27 \\
(1.01-10.59)\end{array}$ & & & & & \\
\hline Drinking history & & 2.19 & 0.34 & & & \\
\hline Yes & Ref & & & & & \\
\hline No & $0.33(0.08-1.43)$ & & & & & \\
\hline Unknown & 0.00 & & & & & \\
\hline Grade & & 0.62 & 0.89 & & & \\
\hline Well differentiated & Ref & & & & & \\
\hline $\begin{array}{l}\text { Moderate } \\
\text { differentiation }\end{array}$ & $0.60(0.15-2.42)$ & & & & & \\
\hline $\begin{array}{l}\text { Poorly } \\
\text { differentiated }\end{array}$ & $0.86(0.27-2.74)$ & & & & & \\
\hline Unknown & $0.73(0.19-2.73)$ & & & & & \\
\hline T stage & & 0.02 & 0.89 & & & \\
\hline T1-T2 & Ref & & & & & \\
\hline T3-T4 & $0.94(0.43-2.08)$ & & & & & \\
\hline N stage & & & & & & \\
\hline NO & Ref & & & & & \\
\hline N1-N2 & & & & & & \\
\hline NX & & & & & & \\
\hline M stage & & 4.42 & 0.11 & & & \\
\hline M0 & Ref & & & & & \\
\hline M1 & $0.64(0.36-1.16)$ & & & & & \\
\hline$M X$ & $2.10(0.95-4.63)$ & & & & & \\
\hline
\end{tabular}

\section{Abstract IDDF2021-ABS-0106 Figure 4}


analysis shows that high HSBP1 may be involved in the regulation of nucleotide excision and repair, spliceosome, proteasome and other pathways (IDDF2021-ABS-0106 Figure 4). The analysis of immune checkpoint results showed that patients with high HSBP1 expression had increased immune checkpoint molecules CLAG3 and TIGIT (IDDF2021-ABS0106 Figure 5).

Conclusions The iron death-related gene HSBP1 may be involved in the occurrence and development of esophageal squamous cell carcinoma and the regulation of immune checkpoints. This study provides a new research direction for future HSBP1-related esophageal squamous cell carcinoma research and treatment targets.

\section{IDDF2021-ABS-0108 ENTEROBACTERIACEAE - DECIPHERING THE CULPRIT GUT BACTERIA CAUSING NECROTIZING ENTEROCOLITIS IN INFANTS}

${ }^{1} J$ odi Woan-Fei Law*, 'Vengadesh Letchumanan, ${ }^{1}$ Hooi-Leng Ser, ${ }^{2}$ Loh Teng-Hern Tan, ${ }^{1}$ Priyia Pusparajah, 'Learn-Han Lee. 'Novel Bacteria and Drug Discovery (NBDD) Research Group, Microbiome and Bioresource Research Strength, Jeffrey Cheah School of Medicine and Health Sciences, Monash University Malaysia, Malaysia; ${ }^{2}$ Clinical School Johor Bahru, Jeffrey Cheah School of Medicine and Health Sciences, Monash University Malaysia, Malaysia

\subsection{6/gutjnl-2021-IDDF.39}

Background Necrotizing enterocolitis (NEC) is a lethal gastrointestinal illness primarily affecting preterm infants, causing inflammation and necrosis of the intestines. It is apparent that the colonization of certain bacteria in the gastrointestinal tract had contributed to NEC pathogenesis. This research aims to investigate the association of gut pathogens from family Enterobacteriaceae and the development of NEC.

Methods A systematic literature search was performed according to the Preferred Reporting Items for Systematic Reviews and Meta-Analyses (PRISMA) guideline across three databases
(Ovid MEDLINE, ProQuest, and Scopus; dated to 31st May 2021). The research was conducted using MeSH terms: ('gut' OR 'intestinal') AND ('Enterobacteriaceae OR 'pathogen') AND ('neonate' OR 'neonatal' OR 'infant') AND 'enterocolitis'. Studies involved the analysis of the gut microbiome of infants with NEC as compared to those of non-NEC/healthy controls were included. Studies that are irrelevant at the title/ abstract level or without experimental intervention (e.g., reviews) were excluded.

Results Twenty-one studies were identified for qualitative analysis, which amounted to 361 NEC cases. The majority of NEC cases were diagnosed in preterm infants, and the gut microbiome was determined by analyzing fecal/intestinal tract samples using microbial culture or culture-independent molecular techniques. The main findings demonstrated a significant increase in abundance of Enterobacteriaceae colonizing the gut of NEC infants than those of controls. Klebsiella spp. (Klebsiella pneumoniae, Klebsiella oxytoca), Shigella dysenteriae, Escherichia coli, and Citrobacter koseri were identified as the dominating taxa present in the gut of NEC infants (IDDF2021-ABS-0108 Figure 1. Enterobacteriaceae colonizing the gut of NEC infants). However, some studies reported contradicting results due to the inability to pinpoint clear signatures of gut microbial composition causing NEC. Dysbiosis of the gut microbiota caused by a higher abundance of Enterobacteriaceae can be a factor triggering an inflammatory response in premature infants. Preterm infants have immature intestinal host defenses that could expose them to a higher risk of intestinal diseases.

Conclusions The outcome of this systematic review revealed an important trend of increased abundance of intestinal Enterobacteriaceae in NEC. Nevertheless, the role of Enterobacteriaceae in the development of NEC warrants further investigation. A better understanding of NEC is crucial for effective prophylactic and therapeutic interventions.

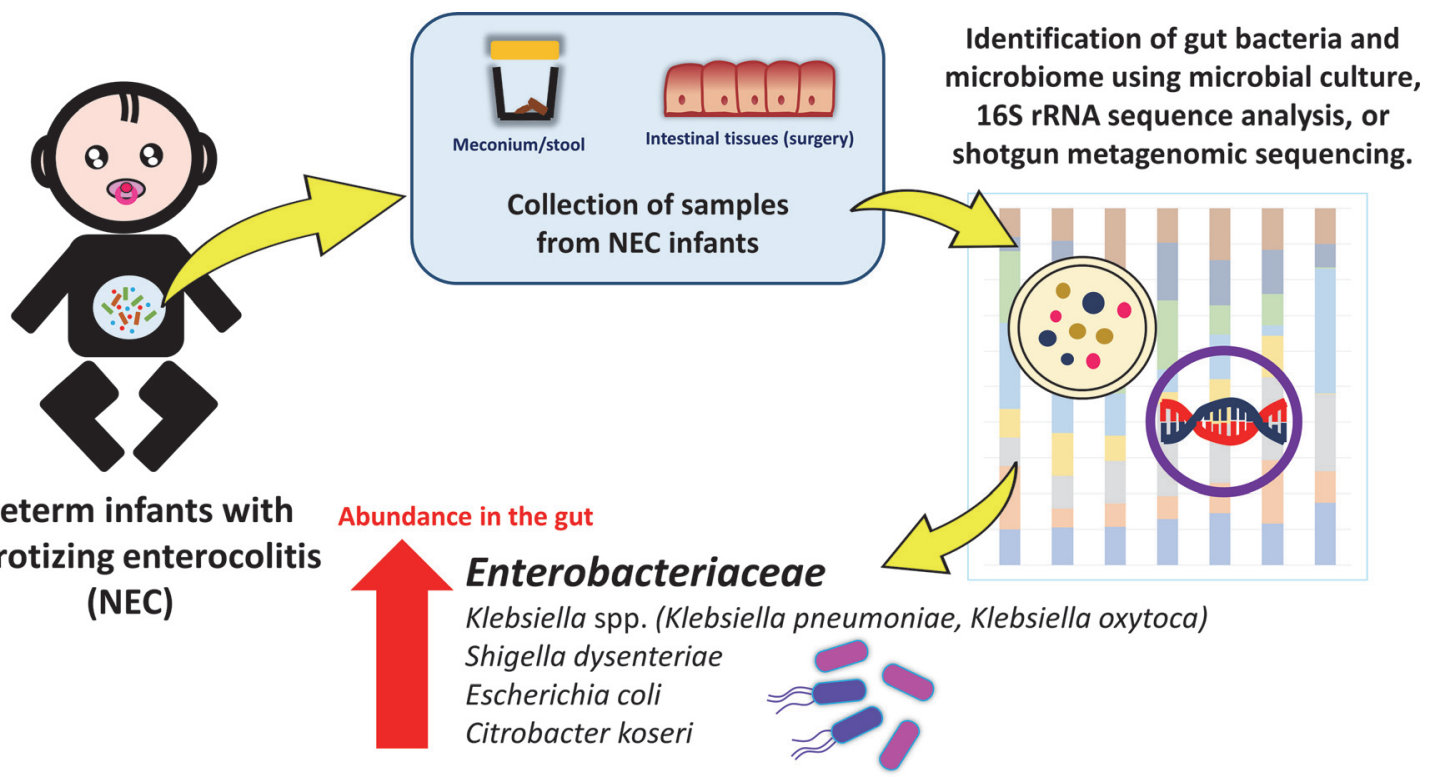

\section{Abstract IDDF2021-ABS-0108 Figure 1}

